Trivent Publishing

(C) The Authors, 2016

Available online at http://trivent-publishing.eu/

Engineering and Industry Series

Volume Power Systems, Energy Markets and Renewable Energy Sources in

South-Eastern Europe

\title{
Information System Supporting the Local EIC Issuing Office
}

\author{
Ivan Fućek, ${ }^{1}$ Boris Golub, ${ }^{1}$ Đordana Miličić, ${ }^{2}$ Nela Bilčar ${ }^{2}$ \\ ${ }^{1}$ Market and extended real-time applications, KONČAR - Power Plant and Electric \\ Traction Engineering Inc., Croatia, \\ ivan.fucek@koncar-ket.hr, boris.golub@koncar-ket.hr \\ ${ }^{2}$ Market activities support section, Croatian Transmission System Operator Ltd., \\ Croatia,djordana.milicic@hops.hr, nela.bilcar@hops.hr
}

\begin{abstract}
This article gives an overview of an information system which supports the standard operation processes of a local issuing office including data maintenance of EIC (Energy Identification Coding) objects and data synchronization with the central issuing office.

Although well-defined, EIC regulations are subject to change. Special attention will be given to recent changes in EIC model definition, local issuing office responsibilities and data exchange workflow as well as their impact on existing information systems.

The first part of the article describes business processes according to the Energy identification coding scheme (EIC) reference manual with a description of recent regulation changes.

This description will include object types which are uniquely identified using the Energy identification coding scheme (EIC), issuing a new EIC codes workflow and the data synchronization process with the Central Issuing Office (CIO). In addition to the implementation guide, the process of providing data to other systems will also be described, thus showing the importance of stored information for other business processes.
\end{abstract}

This is an Open Access article distributed in accordance with the Creative Commons Attribution Non Commercial (CC-BY-NC-ND 4.0) license, which permits others to copy or share the article, provided original work is properly cited and that this is not done for commercial purposes. Users may not remix, transform, or build upon the material and may not distribute the modified material (http://creativecommons.org/licenses/by-nc/4.0/) 
The second part of the article gives an overview of the information system architecture as well as its integration with other systems. The information system for the local issuing office architecture is described through all the layers -- starting from the data layer, then the layer of business logic implemented using the Java platform and data display layer implemented using modern JavaScript frameworks on a Java Enterprise Edition containers. The information exchange with the Central Issuing office in the process of synchronization through the Enterprise Service Bus platform is described, together with the integration with other information systems.

More details about the modular structure of the information system will be given, with stress on achieving high availability of the information system, as well as different options available when deciding which system components are used to implement on and run the information system.

\section{Keywords}

Energy markets; economics and legislation; information system; portal; java

\section{Introduction}

Keeping records of the entities participating in the electric grid market include maintaining data about market participants, resource objects, measurement points, tie-lines etc. The standardization of the identification of these entities led to the need to organize an office which would issue and maintain these identifications. The EIC (Energy Identification Coding scheme) code is a unique code assigned to objects participating in the European electricity grid market. These codes are issued by issuing offices approved by the ENTSO-E organization. In Croatia, the responsibility of organizing such an office belongs to the Croatian's Transmission System Operator.

\section{System Functions}

The market management system (MMS) supports many business processes in the jurisdiction of a transmission system operator. Some of them include: Interconnection Capacity Allocation and Nomination, System Scheduling, Reserve Resource Process, Settlement Process etc. What is common to the above-mentioned systems is the need for a basic set of data including market participants and other grid objects involved in a number of processes. For example, in the process of interconnection allocation and nomination, the way the participants' roles change over time needs to be monitored. A participant may lose the right to participate in a certain auction process in case there is an unsettled financial debit to the transmission system operator. 
In the following sections, specific functions of the system supporting local issuing functions will be presented as well as its integration with other information systems.

\section{A. EIC Codes}

The European unique identification scheme was agreed upon in 2002 as a result of the work of ENTSO-E (European Transmission System Operators) EDI task force (Electronic Data Interchange) [2]. The EIC format is based on the IEC 62325 series (Framework for energy market communication). The IEC 62325450 methodology was applied in order to develop the conceptual and assembly model. EIC codes are widely used for reporting transaction and fundamental data on the ENTSO-E Transparency Platform and the ACER Information System (according to the EU 543/2013 and EU 1348/2014). Up to this day, more than 5000 Market Participants and Data Providers are registered. The following objects are included in the identification process:

- Parties: System Operators, traders, producers, consumers, power exchanges, grid operators, suppliers, agents, service providers, etc.

- Areas: Local grids where metering points are situated, market balance areas consisting of a number of local grids, control areas, etc.

- Measurement Points: cross border connections, settlement or accounting points, etc.

- Resource objects: The objects which generate or consume energy.

- Tie-lines: The physical lines which connect two Market Balance Areas.

- Locations: The physical or logical places where a party or the IT system of a party is or could be located.

In order to keep the record consistent over time, several conditions and rules must be satisfied. Issued identification codes must be unique and only one identification code can be assigned to one object. Once the code is assigned to an object, it can only be modified when the status of an object changes, not if the object's basic data is changed - such as the name or address. Only system operators, market operators and imbalance settlement responsible organizations may request new area identification codes.

The EIC code is comprised of a set of 16 characters. The code can only contain numbers (0-9), capital letters (A-Z) and the minus (-) sign. The code consists of 4 segments. The first 2 characters contain the identification of an area in jurisdiction of a local issuing office (e.g. "31" for Croatian regulation area), while the next character contains the type of an object (e.g. " $\mathrm{X}$ " for market participant). The available characters which define the object type are as following:

- "X" for Market participants;

- "Y" for Areas;

- "Z" for Measurement point;

- "W" for Resource objects; 
- "T" for Tie-lines;

- "V" for Locations;

- "A" for Substations.

The next 12 characters uniquely identify an object. The last character is a check character calculated from the first 15 characters; it may contain numbers, capital letters, but not the sign minus. For example, the following are valid (and in use) EIC codes:

- 31X-HEP-ODS----6 (Croatian Distribution System Operator, significant code)

- 10X1001A1001A248 (Danish Transmission System Operator, insignificant code)

According to the implementation guide, using insignificant codes is strongly recommended.

\section{B. The Local Issuing Office (LIO)}

The Local Issuing Office (LIO) operates as a branch office of the Central issuing office. Every area has the right to establish such an office; in Croatian regulation, an area local office is a department under the Croatian Transmission System Operator Ltd. EIC codes can be assigned to objects related to domestic processes (e.g. measuring point inside regulation area), as well as to objects related to international processes (e.g. international tie-line). Every local issuing office is responsible for maintaining a consistent set of EIC codes assigned to object under its jurisdiction. In addition, every data change of international EIC objects must be reported to the central issuing office. ENTSO-E defines messages for data synchronization between the local and central issuing offices by using "The energy identification coding scheme (EIC)" reference manual. The information system's data model is implemented according to the manual as well as the data synchronization mechanism. The system's user interface is shown in the image below (Fig. 1).

The standard data model is extended in order to support additional information. Additional data are available to the system users as well as to other systems, but are excluded from the synchronization process. 

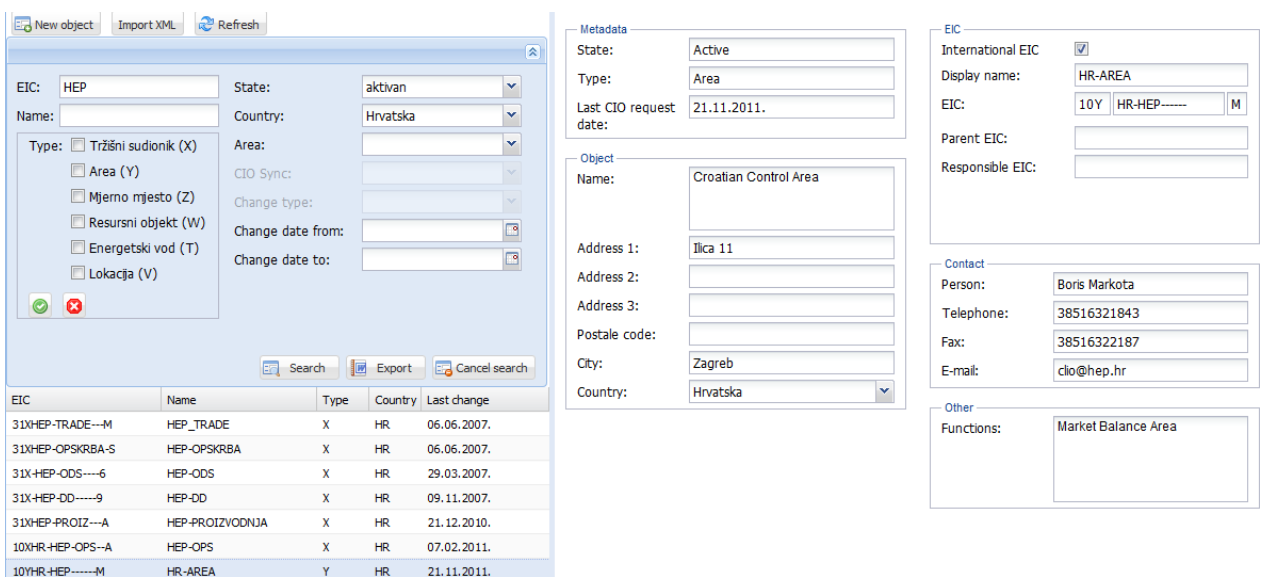

Fig. 1. EIC Object records

\section{The Central Issuing Office (CIO)}

The Central Issuing Office collects data from all local issuing offices. During the submission of the new objects, or the modification of the existing ones, the central issuing office is responsible to keep all data consistent at all times. CIO is also responsible for assigning local issuing office identification codes which are part of every EIC code [4][5]. The central issuing office is responsible for keeping record of all the objects not in the jurisdiction of any local issuing office. The collected data are partially published on a public web site, while the local issuing office has access to all the data stored in a central repository.

\section{The Allocation of EIC Codes and Synchronization with CIO}

The information system supports a two-way communication with the central issuing office. Communication takes place in any of the following situations:

- Allocation of new EIC codes for new objects

- Object data or status change

- Central repository is updated

In case of assigning a new EIC code, the system supports manual EIC code entry from a user as well as automatic EIC code generation based on its characteristics (object type, local issuing office identification code etc.). After new data are inserted or existing ones are changed, the system changes the object's status which describes the object state of synchronization. As a result, the system can collect all the data that are changed and automatically generate the request message which has to be sent to the central issuing office. The message generated is in .xml format according to the manual. The user can review all the changes from the user interface, as shown on the image below (Fig. 2). 

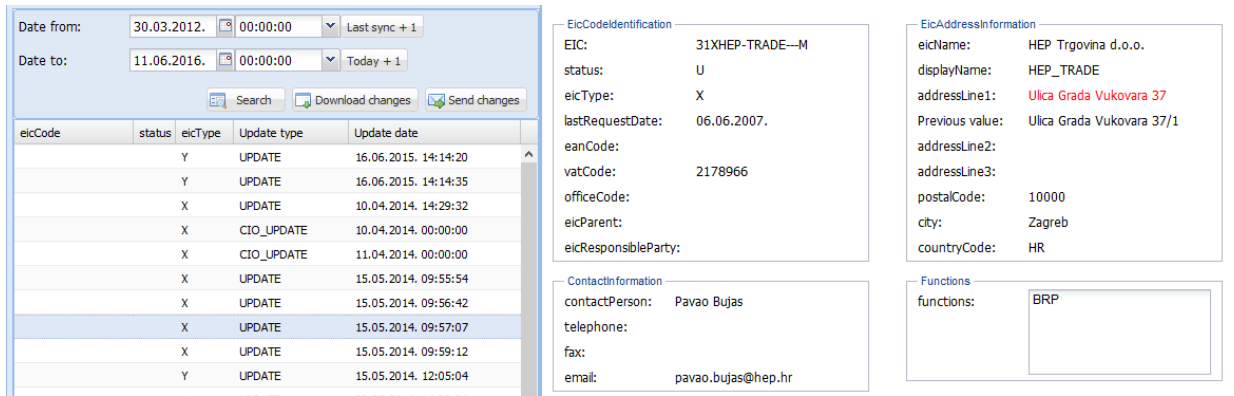

Fig. 2. Overview of modified Objects

The system is able to show the user when the object was changed, and is able to colour fields that were changed based on the values before the change. After the user selects all the changes from the last synchronization point and generates the request, the system changes the status of the selected objects to show that these changes are sent to the central issuing office. After the central issuing office replies to the request, the reply message can be directly uploaded to the system. During the upload, the system analyses the reply and edits the data accordingly. If some of the changes are not approved by the central issuing office, they would be changed in the response. System recognises these changes and edits the data in the local repository accordingly. After the response is fully uploaded, all the data in the local repository is synchronized. The process described can be applied when adding new EIC objects, modifying an existing one, or when activating, deactivating or reactivating an object. The synchronization steps [3] can be seen on the image below (Fig. 3).

The system supports automatic communication with the central issuing office by e-mail (messages are transferred in body or attachment). If another means of communication is necessary, the user is enabled to download a generated message and send it by using the appropriate communication channel and upload the response afterwards. In case of data change from another local issuing office, the system supports the upload of the entire central issuing office repository. 


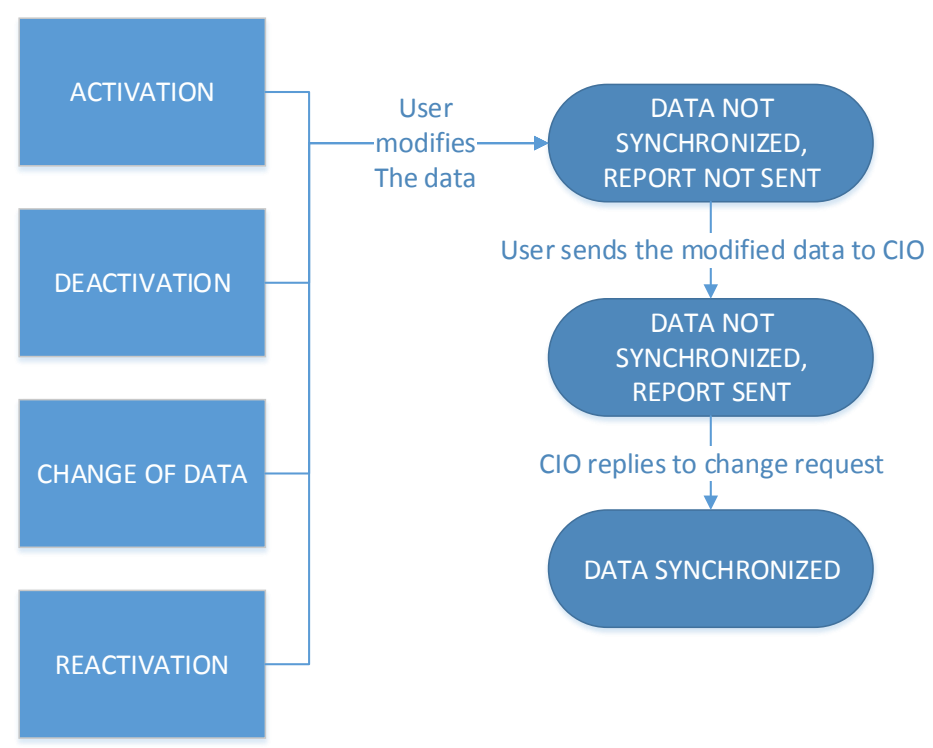

Fig. 3. The steps of the Data Synchronization Process

\section{E. System Integration}

Repository data are available to other systems via web service technology. Other systems can use all the data as a basic set of information that can be extended according to the implemented business processes.

\section{System Architecture}

The architecture is based on the standard three-tier architecture model usual for applications compiled in Java software language. The three-layer architecture model is a client-server architecture consisting of a layer of business process data display, a layer of business logic and a layer of data repository. Individual layers are organized as logically-separated units. Dividing an application into separate units enable changing (or replacing) an individual layer, while others can remain the same thus simultaneously increasing the flexibility, sturdiness and usability of the system.

\section{A. The Presentation Layer}

The presentation layer is the highest application layer from the logical point of view. It shows data obtained from the communication with the business logic layer and is based on two technologies: JSP and ExtJS.

JSP (Java Server Pages) is a standard JEE (Java Enterprise Edition) technology for application web user interface. ExtJS is a software function library in JavaScript programming language that enables the compilation of very 
rich web interfaces. ExtJS is an implementation of the technology that enables the compilation of Web 2.0 applications. ExtJS also includes multi-browser and multi-lingual support. The user interface based on ExtJS uses REST (Representational State Transfer) communication protocol for accessing the business logic layer. The REST protocol also simplifies the unit testing thus supporting faster application development. The data transfer is based on the JSON (JavaScript Object Notation) standard for Web 2.0 applications. JSON is a concise notation that includes the transmission of a large quantity of meta-data thus reducing the quantity of network traffic between the client workstation computer and the server - meaning a faster application operation as a final consequence.

The presentation layer always accesses data exclusively from the business logic layer - there is no direct access to data stored in the data storing layer or a mechanism enabling it. The implementations based on the afore-mentioned JSP standard enable deployments on various platforms including IBM WebSphere, Oracle, JBoss etc.

\section{B. The Business Logic Layer}

The business logic layer controls the application functionality. This layer is implemented using the SOA (Service Oriented Architecture) guidelines [1] programme modules are implemented as services. Those services are exposed to other applications enabling simpler integration with other information system modules and applications. The implementation of this layer uses the Spring framework that enables the simple compilation of applications using smaller components. Each component is a Java class comprising only certain business logic that can be used again in a completely different business context.

All the other functionalities such as transaction controls, users log in and log out, audit, and access control are added to the application through the spring configuration. Providing services to other information system parts is realized in the form of SOAP web services. XML messages independent of the platform are used to exchange messages over web services. JPA (Java Persistence API) is used for access to the data store layer. JPA enables Java object mapping to the database objects (tables and views). JPA is only a specification, while the specific implementation used in the application is Hibernate [3]. Spring completely supports JPA and Hibernate and simplifies applications which use these technologies. By using JPA, the application becomes independent of the used underlying database. JPA adjusts the application and the SQL queries automatically in accordance to the used database.

The business logic layer runs on Java application server runtime environment (IBM WebSphere Application Server running WebSphere Portal Server in the specific case of the Croatian TSO). The IBM WebSphere Portal is a set of software tools which enables building and managing web portals. It was primarily used to provide a single access point to web content and applications, 
while delivering differentiated and personalized experiences to each user, as well as reliability and scalability in clustered environment.

\section{The Data Layer}

The data store layer consists of a relational database with the role of keeping the independency of data with respect to application servers and the business logic. The separation of this layer increases the scalability and the performance of the application. Data integrity and safety is also assured within the database layer. The application uses the Oracle database, but due to using the Hibernate technology, all modern relational bases supported by Hibernate are also supported.

\section{System Integration}

Repository data are available to other systems via the web service technology implemented in the Java programming language. The access to the web service is enabled for other systems independent of its language implementation as long as they support the SOAP (Simple Object Access protocol) based communication. The web service implementation enables access control and allows connection only to authorized users or systems. The communication with the Exchange mail server is achieved as an independent application developed for the IBM Message Broker platform as an implementation of Enterprise Service Bus (ESB).

\section{Conclusion}

Before the system was implemented, a couple of main goals were defined. The first one was to implement a central repository of fundamental data that would be easily accessible to other existing systems, as well as to the systems that will be implemented in the future. The second one was to develop an information system whose modules could be easily modified in order to deploy on different platforms and environments. By using the Java language, the system as a software can be deployed to various environments, from simple, single instance application servers for either a small or a large number of users, redundant and highly available platforms common to enterprise usage.

As described, the system fully supports the local issuing business processes according to the implementation manual as well as some extended functionalities to meet the needs of the end user. In practice, every business process is subject to change. With a minor effort, the information system can be modified to implement changes in the data model as well as the communication with other systems. 


\section{References}

Papers from Conference Proceedings:

[1] I. Janeš, B. Golub, "Information systems across the energy utility based on Service Oriented Architecture" in 30. International Convention on Information and Communication Technology, Electronics and Microelectronics, Opatija, 2007

Standards:

[2] The Energy Identification Coding Scheme (EIC) Reference Manual Version 5.1

[3] ENTSO-E EIC Data Exchange Implementation Guide Version 1.0

[4]LIO ENTSO-E (10) Energy Identification Code (EIC) Management Version 2.0

[5] Central Issuing Office (CIO) Procedure For The Acceptance Of New Local Issuing Offices Version 1.0

[6] ENTSO-E Harmonised Role model

[7]ENTSO-E Code list

[8]ENTSO-E, Implementation Guide, Acknowledgment Documents Version 5, Release 0

\section{Biographies}

Ivan Fućek is a system engineer at the KONČAR - Power Plant and Electric Traction Engineering Inc., Zagreb. He earned his master's degree in computing engineering at the Faculty of Electrical Engineering and Computing (FER), Zagreb in 2011.

His employment experience includes developing customized software for operators in the electrical power business: Croatian transmission system operator Ltd. (HOPS) and Croatian Energy Market Operator (HROTE). The main features of the developed software are safety, reliability and high level of customization. In addition to his developer skills, Ivan participated in other phases of software development like planning, coordination and presentation.

Boris Golub is the head of the IT sector at the KONČAR - Power Plant and Electric Traction Engineering Inc., Zagreb. He earned his master's degree in Computer Science and Mathematics at the Department of Mathematics, Faculty of Science, in Zagreb, 2003.

His employment experience included working with the Croatian software development companies MIT Software Ltd. and KONČAR - Power Plant and Electric Traction Engineering Inc. in different job positions starting from development engineer, business analyst to project management.

His main references include the development of numerous Market Management System modules for the Croatian Transmission System Operator. During his working experience in KONČAR, he was the co-author and author of 
several technical articles dealing with information system implementation efforts covering different parts of the industry business roles and processes.

Nela Bilčar graduated in 1983, from the Faculty of Electrical Engineering and Computing in Zagreb.

After graduation, she started working for the Croatian Electricity Utility (HEP) where she gained a wealth of experience in development and maintenance of IT systems which support business activities of the transmission system operator. She coordinated the adaptation of the National Dispatch Centre's IT systems during the reconnection of the $2^{\text {nd }}$ synchronous zone to the main UCTE (Union of Co-ordination of Transmission of Electricity) grid. During the past 10 years she has led projects aiming the harmonization of software systems with the requirements of the Croatian and European regulations.

Since July 2012, she has been working for the Croatian Transmission System Operator Ltd. (HOPS). A special area of her interest is the development of the information systems that support the activities of transmission system operator in the electricity market.

She has been actively participating in the work of HRO CIGRE as an author and co-author of technical articles and as lecturer at seminars.

Đordana Miličić is the head of the Market Activities Support Section within the Process and Business IT and Telecommunications Department at the Croatian Transmission System Operator Ltd. (HOPS). She obtained her master's degree in Computer Science at the Department of Applied Mathematics, Faculty of Electrical Engineering and Computing (FER), in Zagreb in 2002.

After graduation, she started working for the Croatian Electricity Utility (HEP) where she gained a wealth of experience in the preparation of business requirement specifications, development and maintenance of IT systems that support business activities of the transmission system operator.

She is a member of the IEC TC57 WG 16 and ENTSO-E working groups dealing with contextual and assembly models for the European electricity market, electronic data interchange and market information transparency. In addition, she is HOPS's Transparency Platform Coordinator (TPC) for the ENTSO-E Transparency Platform. TPC, on behalf of the respective TSO, facilitates the maintenance of the appropriate information related to TSO power systems and in coordination with neighbouring TSO's TPC requests from CIO creation or update of EIC codes (10X, 10Y, 10T and 10Z codes).

She participates in the work of HRO CIGRE by being an author and coauthor of technical articles and by lecturing seminars. 